\title{
Intelligent Building Load Scheduling Based on Multi-Objective Multi-Verse Algorithm
}

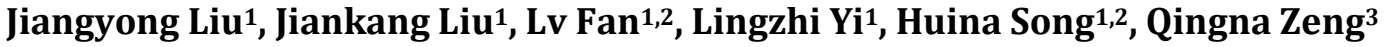 \\ ${ }^{1}$ College of Information Engineering \& Hunan Province Engineering Research Center for Multi-Energy Collaborative Control \\ Technology, Xiangtan University, Xiangtan, China \\ ${ }^{2}$ Willfar Information Technologies Co. Ltd., Changsha, China \\ ${ }^{3}$ Lianhu Town Central Health Center, Chongqing, China \\ Email: Liujiangyong@sina.com,18723940495@163.com,13739588316@qq.com,ylzwyh@xtu.edu.cn,16299972@qq.com, \\ 1948894887@qq.com
}

How to cite this paper: Liu, J.Y., Liu, J.K., Fan, L., Yi, L.Z., Song, H.N. and Zeng, Q.N. (2021) Intelligent Building Load Scheduling Based on Multi-Objective Multi-Verse Algorithm. Energy and Power Engineering, 13, 19-29.

https://doi.org/10.4236/epe.2020.134B003

Received: February 5, 2021

Accepted: April 5, 2021

Published: April 8, 2021

\begin{abstract}
In the multi-objective of intelligent building load scheduling, aiming at the problem of how to select Pareto frontier scheme for multi-objective optimization algorithm, the current optimal scheme mechanism combined with multi-objective multi-verse algorithm is used to optimize the intelligent building load scheduling. The update mechanism is changed in updating the position of the universe, and the process of correction coding is omitted in the iterative process of the algorithm, which reduces the computational complexity. The feasibility and effectiveness of the proposed method are verified by the optimal scheduling experiments of residential loads.
\end{abstract}

\section{Keywords}

Intelligent Building, Load Scheduling, Multi-Objective Optimization, Multi-Objective Multi-Verse Algorithm

\section{Introduction}

Intelligent building is the development direction of future urban residents, realizing automatic load control and automatic demand response. Therefore, the problem of building load scheduling is very important. The electricity situation of the residential side is increasing in the future development [1] [2]. The demand response strategy is implemented to constrain the consumption of electricity on the user side. In other words, the consumption habits of users will change.

When residential users participate in demand response and implement demand side management, load control is carried out according to residents' elec- 
tricity demand and habits. Home electrical loads can be divided into schedulable loads (SLs) and non-schedulable loads (NLs) according to the flexibility of scheduling time in [3] [4] [5] [6]. SLs are the user set a controllable time range for scheduling without causing user dissatisfaction. NLs refer to the load that will greatly cause users' dissatisfaction when transferring power to equipment, and no scheduling is adopted for this kind of load. Therefore, optimizing the running time of SLs is one of the important ways to realize the user-side demand response. SLs can be transferred to a relatively low tariff period within the range of the user's power requirements.

The SLs was controlled to complete the demand response. Demand response includes two categories: price-based demand response (PBDR) and incentive-based demand response (IBDR) in [7] [8] [9] [10]. In the context of demand response based on price, energy scheduling optimization is to explore the energy scheduling of each module in the home micro-grid framework, so that each module can coordinate with each other to meet the basic needs of household energy consumption and achieve different objectives of scheduling optimization. Compared to the price demand response, although the motivational demand response is also in the economic considerations, the mechanism is different. Compared to the price demand response, although the motivational demand response is also in the economic considerations, the mechanism is different. The motivational mechanism mainly encourages users to adjust electricity consumption, balance the time, and ensure the stable operation of power grid, through reward users during peak hours to reduce the load behavior.

The electricity price strategy of market economy occupies a very important position. Price-based demand response is to affect users' electricity consumption patterns and change their inherent energy consumption habits through the electricity price regulation mechanism, so as to reduce the peak and fill the valley and ensure the stable operation of the power system in [11]. The study of PBDR is also divided into time-of-use (TOU), real-time, tiered, fixed and peak prices. The application of TOU tariffs is in line with the current national level of science and technology, while the application of real-time price can't be applied in the general environment. In addition, TOU tariff is combined with ladder electricity price [12]. TOU tariff can fulfill the function of peak clipping and valley filling, while ladder electricity price limits the use of total electricity. The time-sharing electricity price will not change in a short time, so it is very important to study the time-sharing electricity price. The application of TOU tariffs on the residential side is also the trend of development in the future.

\section{Building Resident Model}

\subsection{Schedulable Load Model}

\section{A translational continuous workload model}

Can translation work load can change in the load control time starts running time of load, like electric rice cooker and pot of hot water load can change the 
start time, short time can start time to start originally in a controlled time moment forward or backward shift a certain amount of time, the optimal choice for peak electricity price time running translation to slack electricity run time [13]. This is shown in Figure 1. The translational continuous workload has three times in a controllable period: the earliest start time of the controllable period and the latest start time; the beginning time of the start time of the load operation; the load operation can't be interrupted in the period of the load operation; the load is continuous workload. Where $\mathrm{T}_{\text {on }}$ and $\mathrm{T}_{\text {off }}$ represent the start time and end time of the load that can be translated in the controllable time $T_{1}-T_{N}$, and $T_{\text {turn }}$ represents the time that the load needs to work continuously in this controllable period.

\section{Continuous interruptible load model}

Interruptible load model can work in a controlled time, meet the resident permit of interrupt load operation conditions for short periods of time, and did not cause residents are not satisfied with return to work when the load state of interruptible load working hours work, biggest interruptible load time and load minimum continuous working time, load operation process, the load should not exceed maximum when interruptible time period, after the minimum continuous working time work load can be interrupted operation. As is shown in Figure 2, $\mathrm{T}_{1}, \mathrm{~T}_{2}, \mathrm{~T}_{3}, \ldots, \mathrm{T}_{\mathrm{m}}$ represent the load-controlled continuous working state. The continuous working time reaches the minimum running time of interruptible load $\mathrm{T}_{\min }$, and $\mathrm{T}_{\mathrm{m}+1}$ can exit the working state or continue working at the next moment. $\mathrm{T}_{\mathrm{m}+1}, \ldots, \mathrm{T}_{\mathrm{m}+\mathrm{n}}$ mean that the continuous non-working time of the interruptible load cannot exceed the maximum non-working time. If $\mathrm{T}_{\mathrm{n}}=$ $\mathrm{T}_{\max }, \mathrm{T}_{\mathrm{n}+1}$ must enter the working state at the next moment.

\section{Charge/discharge load model}

Charging/discharging loads, such as electric vehicles, charge during periods of low electricity price, and send electricity to the grid or home energy management system during periods of peak electricity consumption or peak electricity

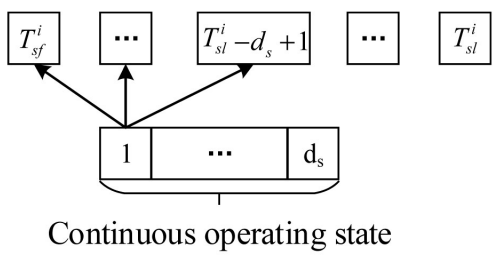

Figure 1. Diagram of translational load scheduling.

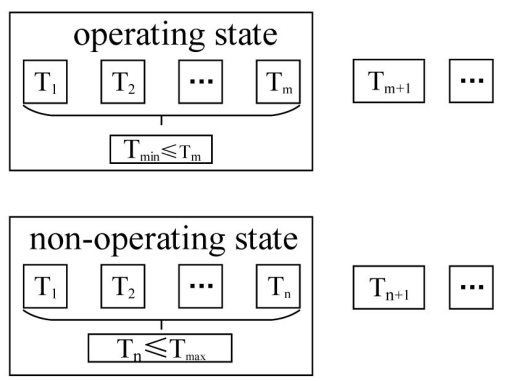

Figure 2. Diagram of continuous interruptible load scheduling. 
price. Electric vehicles charge at night during the day, and send power to the energy management system during periods of peak electricity price during the day, as shown in Figure 3.

Such loads require additional constraints on the amount of battery power, charging according to the grid price, and subsidizing the loss of electric vehicles when delivering power to the home energy management system.

Add constraints for charge/discharge loads:

$$
C_{\text {pre }}+\sum_{j=T_{o n}}^{T_{\text {off }}} \eta_{c h} X_{j} P_{j} \leq C_{\max }
$$

where $X_{j}$ is the load state of charge/discharge load in period $j$ (1: charging, 0 : hanging, -1: discharge). $C_{p r e}, \eta_{c h}, P_{j}$ and $C_{\max }$ represent the initial charge of charging load, charging efficiency, charging power and maximum chargeable amount respectively. $T_{\text {on }}$ and $T_{\text {off }}$ represent the starting and ending time of charging respectively.

\subsection{Additional Energy Storage Load Model of New Energy}

Due to the unstable power of photovoltaic and wind power and other new energy sources, batteries are needed for energy buffer and stable output voltage to be directly supplied to building residents, as shown in Figure 4. Additional energy storage load of new energy (battery is selected in this paper). The battery mainly stores the multi-generation capacity of new energy and supplies power to the building residents at the next peak of electricity consumption or peak price, as shown in Figure 5.

In order to prolong the service life of the battery, additional energy storage load is applied to the battery quantity at the first moment, the maximum and minimum charging/discharging degree, $\alpha_{\max }$ and $\alpha_{\min }$ the constraint conditions.

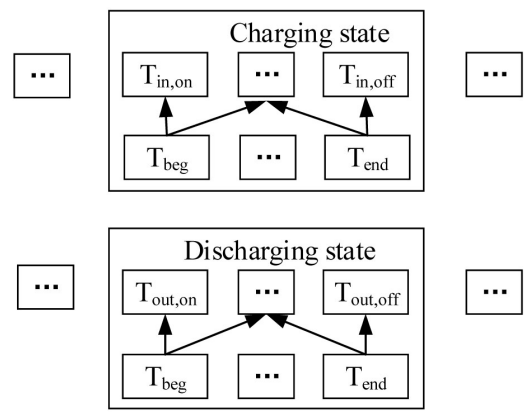

Figure 3. Diagram of charging/discharging load scheduling.

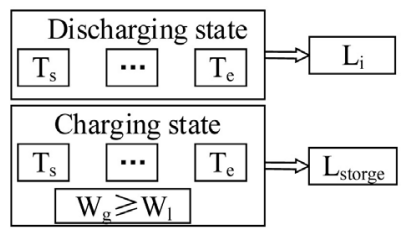

Figure 4. Diagram of new energy power transmission and scheduling. 


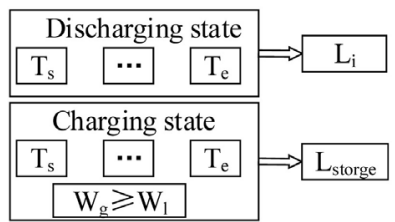

Figure 5. Diagram of energy storage load and scheduling.

$$
C_{h}=C_{p r e}+\sum_{j=1}^{h} \eta_{c h} x_{j} p_{j}+\sum_{j=1}^{h} \eta_{d i s} x_{j} p_{j} \forall h, \alpha_{\min } C \leq C_{h} \leq \alpha_{\max } C
$$

where cap $_{i}$ is the electric quantity of the additional energy storage load of new energy at the moment, $c a p_{\text {pre }}^{i}$ is the initial electric quantity in a day. $\eta_{c h}, \eta_{d i s}$, $x_{j}$ and $p_{j}$ are the charging efficiency, discharge efficiency, the running state at the moment and the charging power respectively.

\subsection{Objective Function of Optimal Scheduling of Building Load}

Under the permissible range of the electrical comfort of building residents, the objective function model is established by comprehensively considering the user side, the grid side and the new energy generation side, and taking the total electricity cost of the user side as the lowest, the stable operation of the grid and the lowest electricity consumption at the peak price, and the lowest power energy discard rate of the new energy side as the lowest.

$$
\begin{gathered}
\min F=[C, D] \\
C=\sum_{i=1}^{n} \sum_{j=1}^{H} T_{i, j} P_{i, j} c_{i, j} \\
D=\sum_{j=1}^{H}\left|E_{j}-\bar{E}\right| \\
\bar{E}=\sum_{j=1}^{H} E_{j} / H
\end{gathered}
$$

where $C$ and $D$ are the total electricity cost (in RMB) of all households in a day and the deviation value $(\mathrm{kWh})$ of electricity consumption respectively, $T_{i, j}, P_{i, j}$ and $c_{i, j}$ represent the period of the first consumption of load $i$, power and TOU price respectively. $n$ is the total quantity of load. $H$ is the number of time periods divided into 24 hours (96 in the paper). $E_{j}$ and $\bar{E}$ represent the power consumption at the $j$ th moment and the average power consumption of a day respectively.

\section{Multi-Objective Multi-Verse Algorithm Based on Intelligent Building Load Scheduling}

\subsection{Multi-Objective Multi-Verse Algorithm}

The multi-verse algorithm and swarm intelligence algorithm (such as particle swarm optimization algorithm and genetic algorithm) are divided into two stages in the iterative process: exploration and exploitation. In the multi-verse algo- 
rithm, a population is one universe, and in one universe there are black holes, white holes and wormholes. White holes and black holes are used to search space for exploration. Wormholes are used for mining search space. Multi-objective multi-verse algorithm and multi-verse algorithm have the same mechanism. The population distribution of MOMVO is such as Equation (7). Where $P$ is population space, $P_{j}$ denotes the $t$ th population. $x_{j}^{k}$ denotes the $k$ th variable of the th population.

$$
P=\left[\begin{array}{c}
P_{1} \\
P_{2} \\
\vdots \\
P_{n}
\end{array}\right]=\left[\begin{array}{cccc}
x_{1}^{1} & x_{1}^{2} & \ldots & x_{1}^{c d} \\
x_{2}^{1} & x_{2}^{2} & \ldots & x_{2}^{d} \\
\vdots & \vdots & \vdots & \vdots \\
x_{n}^{1} & x_{n}^{2} & \ldots & x_{n}^{c d}
\end{array}\right]
$$

In the MOMVO, the population update mechanism and the multi-verse update mechanism are almost as shown in Equation (8). $X_{\text {best }}^{k}$ represents the best universe. $T_{D R}$ is the traveling distance rate, which is calculated as Equation (9). $W_{E P}$ is Wormhole existence probability (WEP), which is calculated as Equation (10). $u_{b}^{k}$ and $l_{b}^{k}$ represent the upper and lower limits, respectively. $r_{a}, r_{b}$, $r_{c}$, and $r_{d}$ are all random numbers $[0,1] . n_{j}\left(P_{j}\right)$ is the normalized expansion rate of the th universe. $W_{E P, \min }$ and $W_{E P \text {, max }}$ are the minimum and maximum values of the wormhole existence rate, respectively. $l$ and $L$ are the current iteration number and the maximum iteration number, respectively. $c$ is the mining accuracy in the iterative process.

$$
\begin{aligned}
& x_{j}^{k}= \begin{cases} \begin{cases}X_{b e s t}^{k}+T_{D R} \times\left(\left(u_{b}^{k}-l_{b}^{k}\right) \times r_{d}+l_{b}^{k}\right) & r_{c}<0.5 \\
X_{b e s t}^{k}-T_{D R} \times\left(\left(u_{b}^{k}-l_{b}^{k}\right) \times r_{d}+l_{b}^{k}\right) & r_{c} \geq 0.5\end{cases} & r_{b}<W_{E P}\end{cases} \\
& W_{E P}=W_{E P, \text { min }}+l \times\left(W_{E P, \text { max }}-W_{E P, \text { min }}\right) / L \\
& T_{D R}=\left(L^{1 / c}-l^{1 / c}\right) / L^{1 / c}
\end{aligned}
$$

\subsection{Multi-Objective Multi-Verse Algorithm Based on Intelligent Building Load Scheduling}

Intelligent building load scheduling is integer programming, so it is necessary to change the initialization of MOMVO. Each variable of the population represents the time when the controllable load starts in a controlled time. In the process of algorithm iteration optimization, it is necessary to ensure that the variable is integer. Applying the original update method, the load code needs to be modified twice. The modification of the population renewal mode is shown in Equation (11). The upper and lower bounds can be automatically satisfied by applying this method, and the diversity of the population during the mining process will not be reduced. 


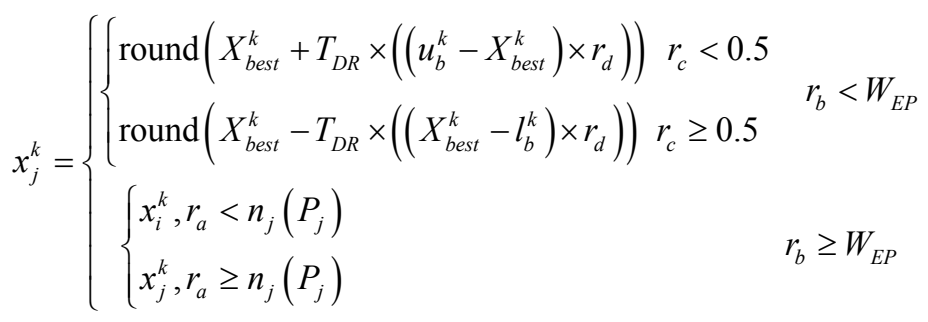

\subsection{Steps and Flow Charts of Multi-Objective Multi-Verse Algorithm}

The steps of solving the model are based on the multi-objective multi-verse algorithm.

Step 1: Import the load forecast electricity consumption data of building residents.

Step 2: Initialize. Initialize according to the upper and lower bounds of each variable. Set the iteration number of algorithm parameters $\mathrm{N}$ and population number $\mathrm{P}$.

Step 3: Calculate fitness. The objective function value is calculated by population variables.

Step 4: Find the optimal universe and inflation rate. The optimal universe and inflation rate were selected after sorting various groups according to the non-dominated method.

Step 5: Population update. Black holes and white holes are explored, while wormholes are mined to complete the update iteration of the population.

Step6: Judge whether the iterative condition is satisfied, the output result is satisfied, and the iteration condition is not satisfied, return to Step 3.

Figure 6 is an iterative flow chart of intelligent building load based on MOMVO.

\section{Results and Discussion}

This experiment uses the electricity load of 120 residents in a certain community. The total electricity consumption of all residents in a day is shown in the

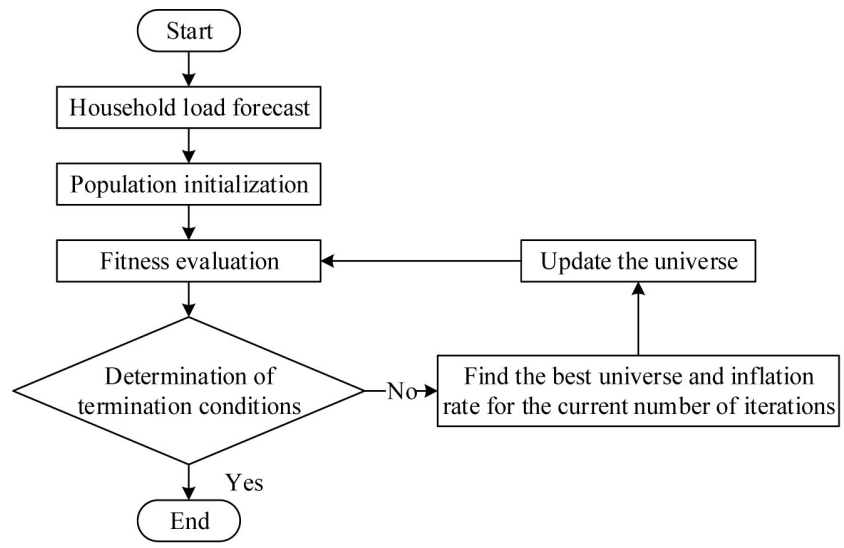

Figure 6. A flow chart of MOMVO. 
Figure 7. When the new energy system is added, the electricity consumption of residents and the electricity supply of the grid are shown in Figure 8. The predictions for renewable energy (wind and photovoltaic) are shown in Figure 9. The algorithm parameters are set as follows: the population number is 300 , the iteration number is $200, W_{E P, \min }$ and $W_{E P, \max }$ is 0.2 and 1 , respectively. The value of $c$ is 6 .

The TOU tariff of the main grid is formulated and announced by the electricity market. The electricity price at each time of the day is as shown in Equation (12) (in RMB)

$$
c_{\text {TOU }}(t)=\left\{\begin{array}{c}
1.1679,9 \leq t \leq 15,18 \leq t \leq 22 \\
0.3958,0 \leq t \leq 7,22 \leq t \leq 24 \\
0.7563,7 \leq t \leq 9,15 \leq t \leq 18
\end{array}\right.
$$

The intelligent building load scheduling is divided into two scenarios for load scheduling. In the first scenario, only residents' load is scheduled, while in the second scenario, intelligent building load is scheduled after the new energy system is added. Figure 10 is a comparison of the total daily electricity consumption of building households before and after the optimal scheduling. Figure 11 is a comparison between before and after the increase of new energy direct supply to building households.

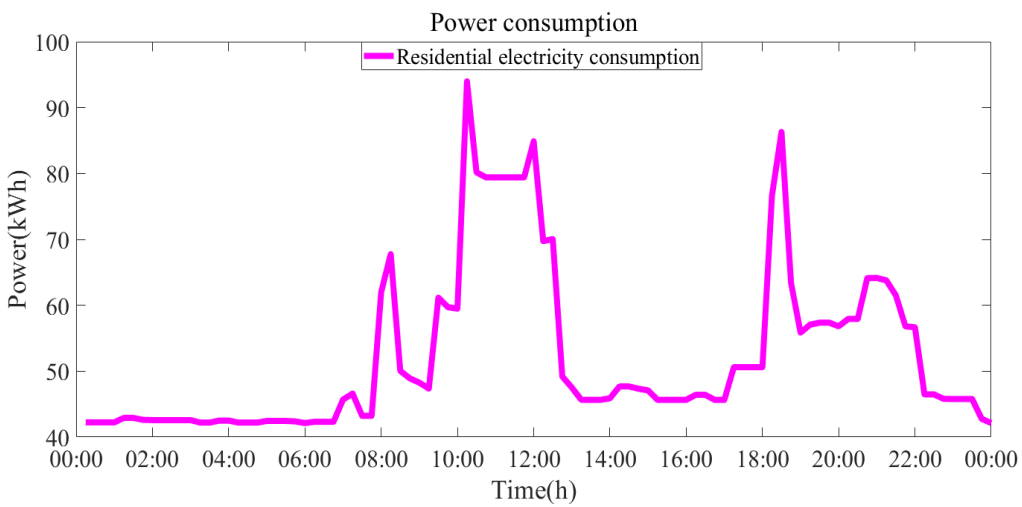

Figure 7. Total electricity demand of building residents.

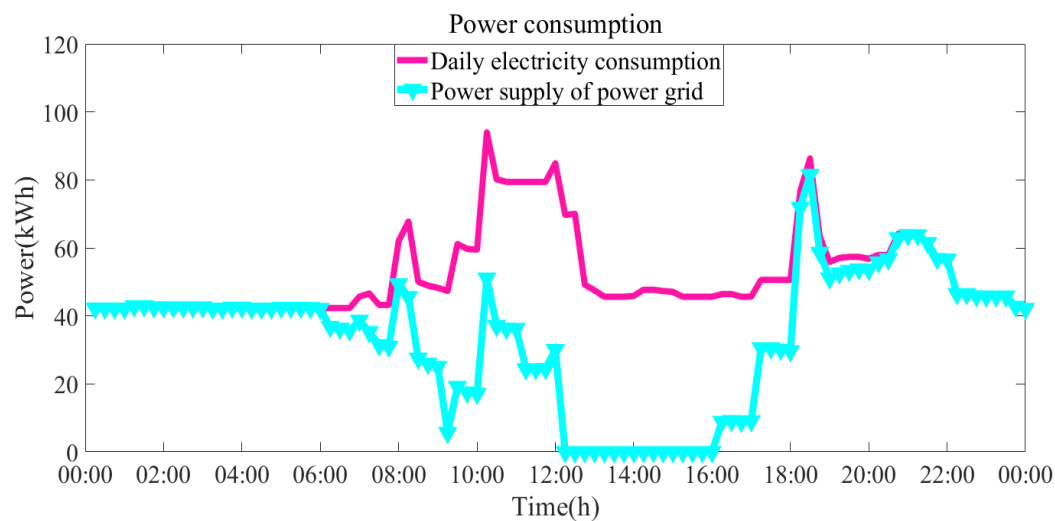

Figure 8. The electricity consumption of residents and the electricity supply of the grid. 


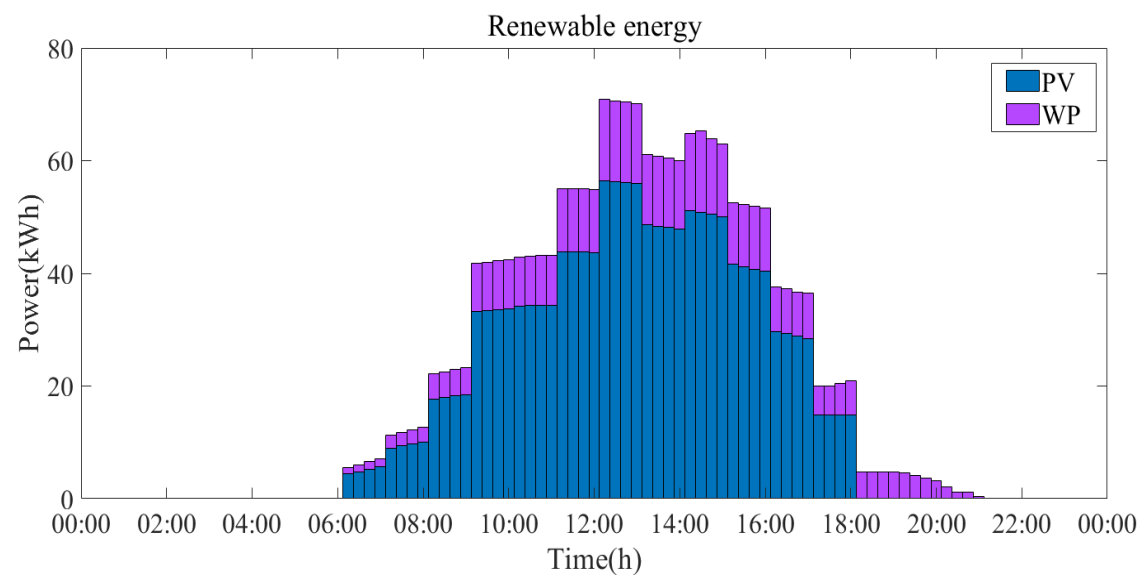

Figure 9. Predicted renewable energy.

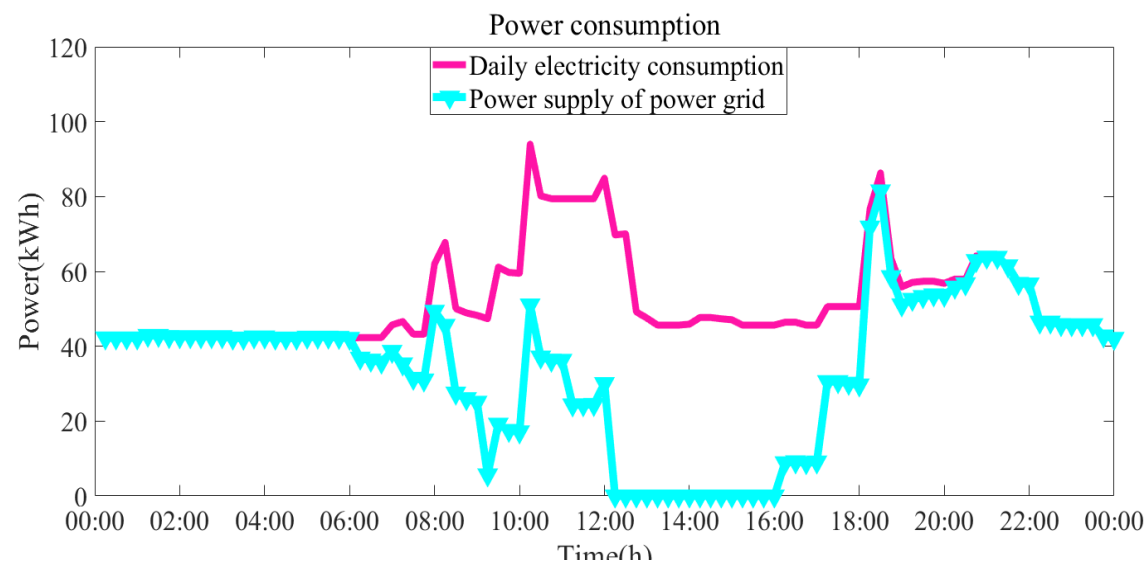

Figure 10. Total daily electricity consumption before and after the optimal scheduling.

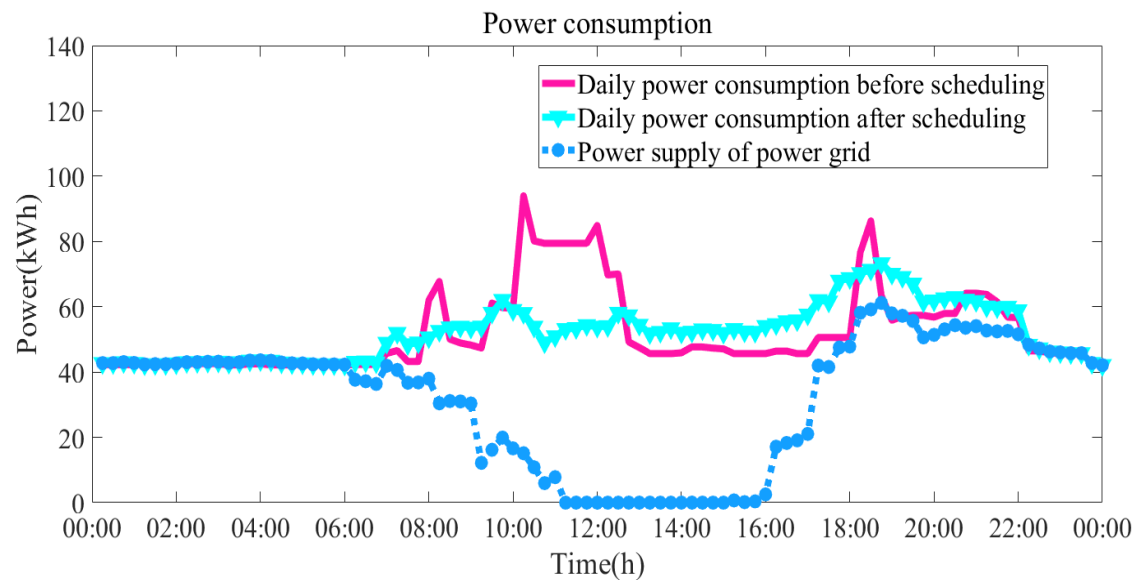

Figure 11. Optimal scheduling of building households with renewable energy system.

Before the optimal scheduling, the resident electricity charge was 4258.9 RMB and the deviation value was $927.6 \mathrm{kWh}$. After the residents' load participated in the scheduling, the electricity fee of the residents was $429.95 \mathrm{RMB}$ and the deviation value of electricity consumption was $590.8 \mathrm{kWh}$. The electricity bill de- 
creased by $65.91 \mathrm{RMB}$, while the deviation value decreased by $337.48 \mathrm{kWh}$. After participating in the scheduling, residents' daily electricity costs are reduced, and the pressure on the power grid is alleviated.

The new energy system joins the scheduling, and the difference between the resident electricity charge and the peak-valley price is $4190.12 \mathrm{RMB}, 589.1 \mathrm{kWh}$. The electricity bill decreased by $68.74 \mathrm{RMB}$, while the difference between peak and valley decreased by $329.52 \mathrm{kWh}$. When renewable energy systems are scheduled, household electricity costs are further reduced and the pressure on the power grid is further alleviated.

\section{Conclusion}

The application of MOMVO to the scheduling of intelligent building residents and the improved update mechanism of MOMVO reduces the correction process and computational complexity of the algorithm in the iterative process. The results show that the time complexity of the algorithm is reduced in the iterative process. The residential load scheduling in intelligent buildings can reduce the daily electricity cost of residents, and the pressure on the power grid can be alleviated. When the renewable energy system is added, the daily electricity cost will be further reduced and the pressure on the power grid will be further alleviated.

\section{Acknowledgements}

This work was supported by the National Natural Science Foundation of China (61572416), Natural Science Foundation of Hunan Province (2020JJ6009), Open subject of The State Key Laboratory of Heavy Duty AC Drive Electric Locomotive Systems Integration and Open subject of The State Key Laboratory of Disaster Prevention and Mitigation for Power Grid Transmission and Transformation Equipment.

\section{Conflicts of Interest}

The authors declare no conflicts of interest regarding the publication of this paper.

\section{References}

[1] Setlhaolo, D., Sichilalu, S. and Zhang, J. (2017) Residential Load Management in an Energy Hub with Heat Pump Water Heater. Appl. Energy, 208, 551-560. https://doi.org/10.1016/j.apenergy.2017.09.099

[2] Pallonetto, F., Oxizidis, S., Milano, F. and Finn, D. (2016) The Effect of Time-of-Use Tariffs on the Demand Response Flexibility of an All-Electric Smart-Grid-Ready Dwelling. Energy Build, 128, 56-67. https://doi.org/10.1016/j.enbuild.2016.06.041

[3] Cui, H. and Zhou, K. (2018) Industrial Power Load Scheduling Considering Demand Response. J. Clean. Prod., 204, 447-460. https://doi.org/10.1016/j.jclepro.2018.08.270

[4] Priya, V., Sathiya Kumar, C. and Kannan, R. (2019) Resource Scheduling Algorithm 
with Load Balancing for Cloud Service Provisioning. Appl. Soft Comput, J., 76, 416-424. https://doi.org/10.1016/j.asoc.2018.12.021

[5] Ahmad, A. and Khan, J.Y. (2020) Real-Time Load Scheduling, Energy Storage Control and Comfort Management for Grid-Connected Solar Integrated Smart Buildings. Appl. Energy, 259, Article ID: 114208. https://doi.org/10.1016/j.apenergy.2019.114208

[6] Zhang, J., Zhu, X., Chen, T., Yu, Y. And Xue, W. (2020) Improved MOEA/D Approach to Many-Objective Day-Ahead Scheduling with Consideration of Adjustable Outputs of Renewable Units and Load Reduction in Active Distribution Networks. Energy, 210, Article ID: 118524. https://doi.org/10.1016/j.energy.2020.118524

[7] Patnam, B.S.K. and Pindoriya, N.M. (2020) Demand Response in Consumer-Centric Electricity Market: Mathematical Models and Optimization Problems. Electr. Power Syst. Res., 106923. https://doi.org/10.1016/j.epsr.2020.106923

[8] Haider, H.T., See, O.H. and Elmenreich, W. (2016) Residential Demand Response Scheme Based on Adaptive Consumption Level Pricing. Energy, 113, 301-308. https://doi.org/10.1016/j.energy.2016.07.052

[9] Zhang, N., Hu, Z., Springer, C., Li, Y. and Shen, B. (2016) A Bi-Level Integrated Generation-Transmission Planning Model Incorporating the Impacts of Demand Response by Operation Simulation. Energy Convers. Manag., 123, 84-94. https://doi.org/10.1016/j.enconman.2016.06.020

[10] Feuerriegel, S. and Neumann, D. (2016) Integration Scenarios of Demand Response into Electricity Markets: Load Shifting, Financial Savings and Policy Implications. Energy Policy, 96, 231-240. https://doi.org/10.1016/j.enpol.2016.05.050

[11] Wang, Y., Lin, H., Liu, Y., Sun, Q. and Wennersten, R. (2018) Management of Household Electricity Consumption under Price-Based Demand Response Scheme. J. Clean. Prod., 204, 926-938. https://doi.org/10.1016/j.jclepro.2018.09.019

[12] Asadinejad, A., Rahimpour, A., Tomsovic, K., Qi, H. and Chen, C.F. (2018) Evaluation of Residential Customer Elasticity for Incentive Based Demand Response Programs. Electr. Power Syst. Res., 158, 26-36. https://doi.org/10.1016/j.epsr.2017.12.017

[13] Yi, L., Liu, J., Yi, F., Lin, J., Li, W. and Fan, L. (2021) Research of Building Load Optimal Scheduling Based on Multi-objective Estimation of Distributed Algorithm. J. Electr. Eng. Technol., 1-12. 